\title{
MÉTODO OPTIMIZADO PARA LA DETERMINACIÓN DE HIDROCARBUROS AROMÁTICOS POLICÍCLICOS ASOCIADOS A PARTIÍCULAS ATMOSFÉRICAS
}

(Optimized method for particulate matter-associated polycyclic aromatic hydrocarbons determination)

\author{
María Florencia TAMES*, Iván TAVERA BUSSO y Hebe Alejandra CARRERAS
}

Departamento de Química, FCEFyN, Instituto Multidisciplinario de Biología Vegetal (IMBiV), CONICET, Universidad Nacional de Córdoba, Córdoba. Argentina, Av. Vélez Sarsfield 1611, Córdoba Capital (CP 5000)

Argentina

*Autora para correspondencia: flor_tames@hotmail.com

(Recibido febrero 2018; aceptado agosto 2018)

Palabras clave: contaminantes urbanos, cromatografía líquida de alto rendimiento, fluorescencia

\section{RESUMEN}

Los hidrocarburos aromáticos policíclicos son contaminantes ambientales ubicuos en el aire, derivados de la combustión incompleta de materia orgánica. Por sus características físicas y químicas, es frecuente encontrarlos asociados a partículas, favoreciendo su permanencia en la atmósfera. En las últimas décadas, el monitoreo de hidrocarburos aromáticos policíclicos en ambientes urbanos se ha convertido en una prioridad debido a la gran cantidad de individuos expuestos. En este trabajo se evaluaron modificaciones de una metodología estándar para la extracción, detección y cuantificación de hidrocarburos aromáticos policíclicos adsorbidos en partículas de $2.5 \mu \mathrm{m}$ de diámetro aerodinámico. Estas variaciones permitieron reducir considerablemente los tiempos y costos de procesamiento, la detección y cuantificación, sin disminuir la sensibilidad para la detección de estos compuestos. Por otro lado, la reducción considerable en la cantidad de disolvente utilizado resulta en una metodología más amigable con el ambiente.

Key words: air pollution, high performance liquid chromatography, fluorescence

\begin{abstract}
Polycyclic aromatic hydrocarbons are ubiquitous air pollutants, derived from the incomplete combustion of organic matter. Due to its physical and chemical characteristics, its association with the particulate matter is favored, and therefore its permanence in the atmosphere. In recent decades, the monitoring of polycyclic aromatic hydrocarbons in urban atmospheres has become a priority due to the high number of exposed individuals. In the present work, modifications of a standard methodology for the extraction, detection and quantification of polycyclic aromatic hydrocarbons were evaluated. These modifications resulted in reductions both in the extraction and quantification time, as well as in processing costs, which implies a substantial improvement in sample processing time, without reducing the sensitivity. In addition, the considerable reduction in the amount of solvent employed resulted in an environmentally friendly methodology.
\end{abstract}




\section{INTRODUCCIÓN}

Los hidrocarburos aromáticos policíclicos (HAP) son contaminantes ambientales ubicuos en el aire, compuestos por dos o más anillos de benceno fusionados, derivados de la combustión incompleta de materia orgánica. Los HAP son térmicamente estables, con puntos de ebullición altos y presiones de vapor bajas, lo que favorece su asociación con las partículas atmosféricas y por lo tanto su permanencia en la atmósfera (Masih et al. 2012).

Estudios previos han demostrado que muchos HAP son mutagénicos y carcinogénicos (Kim et al. 2013) y que su capacidad mutagénica es mayor cuando están asociados a las partículas que cuando están en fase gaseosa (Tsai et al. 1995). En este sentido, los HAP asociados a partículas pequeñas resultan más peligrosos para la salud humana que los adsorbidos en partículas grandes, porque las primeras pueden penetrar más profundamente en el tracto respiratorio y desde allí llegar a otros órganos (USEPA 2003).

En ambientes urbanos, la combustión incompleta de combustibles fósiles en los vehículos es la principal fuente de HAP (WHO 2003). En las últimas décadas, el monitoreo de HAP en la atmósfera se ha convertido en una prioridad en los ambientes urbanos debido a la gran cantidad de individuos expuestos. Más aún, ante la gran cantidad de HAP presentes en atmósferas urbanas, la Agencia de Protección Ambiental de Estados Unidos (USEPA) ha establecido que 16 de estos compuestos son prioritarios para monitoreo debido a su toxicidad (USEPA 2003).

Actualmente existen algunos métodos validados por agencias internacionales para la detección y cuantificación de HAP adsorbidos a partículas, como por ejemplo el establecido por la USEPA para la determinación de estos compuestos en aire utilizando cromatografía de gases/espectrometría de masas (GC/MS) (USEPA 1996). Sin embargo, la mayoría de estas técnicas tienen protocolos muy extensos, complicados metodológicamente y que resultan costosos por la gran cantidad de disolvente que emplean.

En el presente trabajo se evalúan modificaciones de una metodología estándar para la extracción, detección y cuantificación de HAP adsorbidos a partículas que permite acortar significativamente los costos y tiempos de procesamiento de muestras, conservando la sensibilidad y precisión del método.

\section{MATERIALES Y MÉTODOS}

\section{Sitio de estudio}

La ciudad de Córdoba está localizada en el centro de Argentina ( $\left.31^{\circ} 25^{\prime} 00^{\prime \prime} \mathrm{S} ; 64^{\circ} 11^{\prime} 00^{\prime \prime} \mathrm{W}\right)$ y es la segunda ciudad más poblada del país (1.4 millones de habitantes). Tiene una topografía en forma de embudo con una pendiente positiva creciente desde el centro hacia las áreas circundantes. Esta formación un tanto cóncava reduce la circulación de aire y provoca frecuentes inversiones térmicas durante las temporadas de otoño e invierno. El clima es subhúmedo, con una precipitación media anual de $790 \mathrm{~mm}$, concentrada principalmente en verano. La temperatura media anual es de $17.4^{\circ} \mathrm{C}$ y los vientos predominantes provienen del Noreste, Sur y Sureste (Stein y Toselli 1996).

Las principales fuentes de contaminantes atmosféricos están relacionadas con el tráfico y la resuspensión de polvo ambiental (López et al. 2011, Carreras et al. 2013). La ciudad también cuenta con importantes fuentes estacionarias, tales como industrias metalúrgicas y metalmecánicas, localizadas en áreas periféricas al casco urbano (Fig. 1) (Mateos et al. 2018).

\section{Muestreo y determinación de masa de partículas de $2.5 \mu \mathrm{m}$ de diámetro aerodinámico $\left(\mathbf{M P}_{2.5}\right)$}

Se recolectaron dos muestras diarias de partículas $\mathrm{MP}_{2.5}$, utilizando impactadores Harvard con un flujo de aire de entre 16 y $12 \mathrm{~L} / \mathrm{min}$, y un punto de corte esperado de algo más de $2.5 \mu \mathrm{m}$. Se utilizaron filtros de politetrafluoroetileno de $47 \mathrm{~mm}$, con un poro de $2.0 \mu \mathrm{m}$ (Zefluor, Millipore). Uno de los impactadores se empleó para la determinación de la masa, mientras que el otro se empleó para la cuantificación de HAP. Los muestreadores fueron colocados a $7 \mathrm{~m}$ de altura del suelo, en la terraza del Departamento de Química en la Facultad de Ciencias Exactas, Físicas y Naturales de la Universidad Nacional de Córdoba ( $31^{\circ} 26^{\prime}$ 10.9" S; 64 11' 38.8" W), en la ciudad de Córdoba, desde agosto hasta octubre del año 2014. En total se recolectaron 92 filtros para cada determinación.

La masa de $\mathrm{MP}_{2.5}$ fue determinada por diferencia gravimétrica con una microbalanza (Precisa XR205SM-DR, Dietikon, Switzerland). La concentración de $\mathrm{MP}_{2.5}$ fue expresada como la media \pm desvío estándar (DE).

\section{Extracción}

Los filtros conteniendo partículas fueron colocados en tubos de reflujo cerrado (Hach, \#2275800), previamente acondicionados de la siguiente manera: 


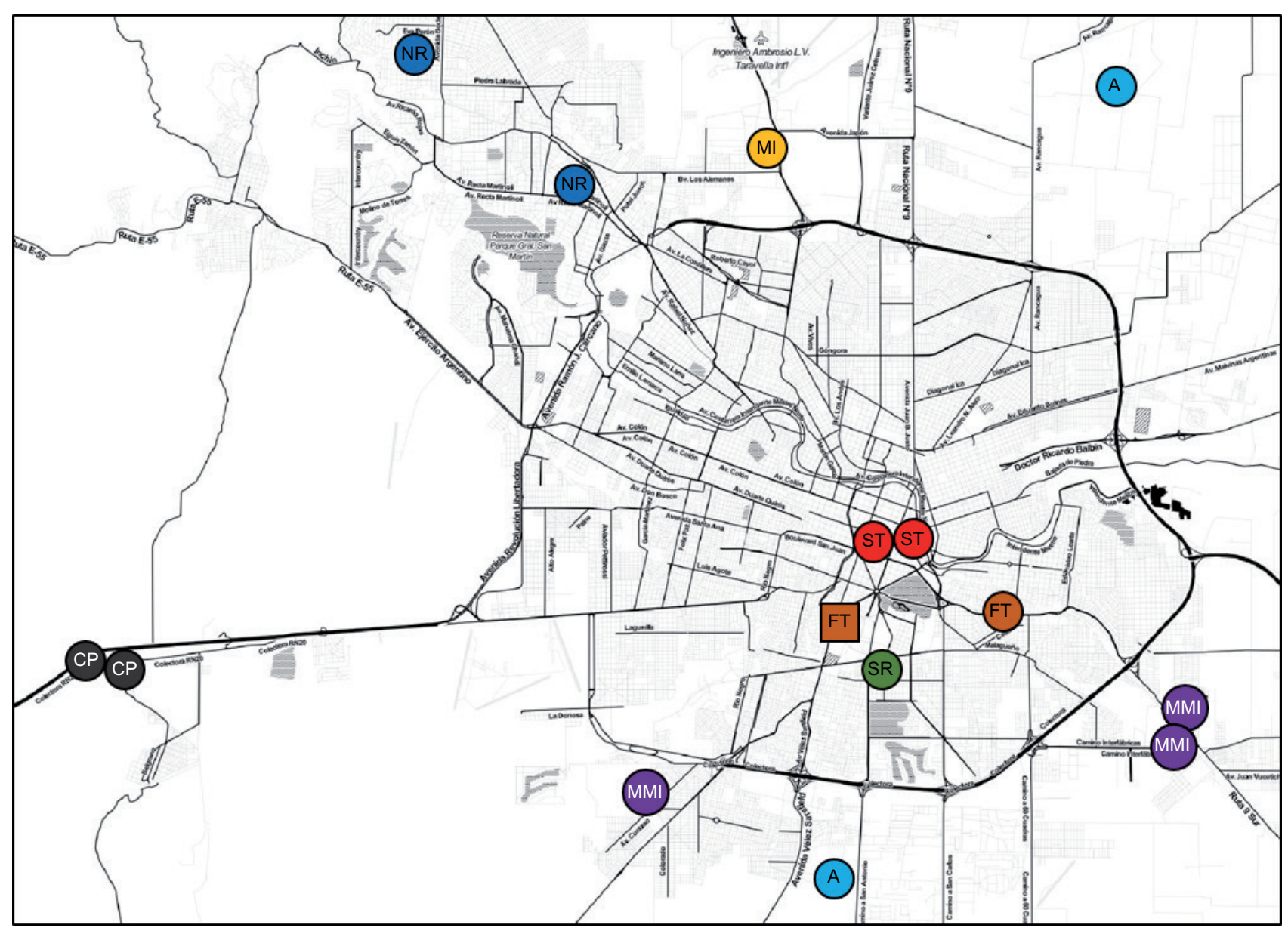

Fig. 1. Ubicación de las principales fuentes de emisión de contaminantes atmosféricos en la ciudad de Córdoba. $\mathrm{A}=$ agricultura, $\mathrm{CP}=$ planta de cemento, $\mathrm{MI}=$ industria metalúrgica, $\mathrm{MMI}=$ industria metalmecánica, $\mathrm{FT}=$ tráfico fluido en avenidas, $\mathrm{ST}=$ tráfico lento en el centro (Mateos et al. 2018). El cuadrado indica el punto de muestreo en este trabajo

se lavaron con agua corriente y detergente no iónico, se enjuagaron con etanol al $96 \% \mathrm{v} / \mathrm{v}$, luego con agua destilada y finalmente fueron llevados a sequedad en una mufla a $450^{\circ} \mathrm{C}$ por $5 \mathrm{~h}$. Previo a su uso, se enjuagó todo el material con el disolvente de extracción. A cada tubo de reflujo cerrado se añadieron $7 \pm 1 \mathrm{~mL}$ de diclorometano (DCM). Dichos tubos fueron posteriormente cerrados herméticamente y se les aplicó ultrasonido (Testlab, Argentina) a temperatura ambiente por 30 minutos y mezclados con vórtex por un minuto, repitiendo estos últimos dos pasos una vez más. El sobrenadante fue trasvasado cuantitativamente a un nuevo tubo de reflujo cerrado con enrase en un $\mathrm{mL}$. El contenido de este último fue concentrado por evaporación de disolvente bajo una corriente de aire filtrado hasta volumen menor a un $\mathrm{mL}$, en tanto que el tubo con el filtro fue nuevamente llenado con DCM como se explicó anteriormente. Este ciclo de extracción y concentración fue repetido tres veces. A continuación, se realizó un cambio de disolvente en el tubo concentrado. Para ello, se añadieron pequeñas porciones de acetonitrilo (ACN) grado HPLC (Pharmco-Aaper, Brookfield), volatilizando, bajo corriente de aire filtrado, el exceso de DCM. Finalmente se procedió a enrasar el contenido a volumen final de un $\mathrm{mL}$ con $\mathrm{ACN}$ de igual calidad.

\section{Composición de HAP}

Se determinaron las concentraciones de naftaleno (Naf), acenafteno (Ace), fluoreno (Flu), fenantreno (Fen), antraceno (Ant), fluoranteno (Fla), pireno (Pir), benzo(a,h)antraceno $(\mathrm{B}(\mathrm{a}, \mathrm{h}) \mathrm{a})$, criseno (Cri), benzo(b)fluoranteno $(\mathrm{B}(\mathrm{b}) \mathrm{f})$, benzo(k)fluoranteno $(\mathrm{B}(\mathrm{k}) \mathrm{f})$, benzo(a)pireno (B(a)p) y dibenzo(a,h)antraceno (Dib(a,h)a). Para ello se empleó un cromatógrafo líquido de alta eficiencia (HPLC por sus siglas en 
inglés, Dionex Ultimate 3000, Thermo Scientific), con una columna C-18 de fase reversa (Vydac 201TP $250 \mathrm{~mm} \times 2.1 \mathrm{~mm}$ i.d., $5 \mu \mathrm{m}$ ) y detector de fluorescencia (FL). Para la curva de calibración se utilizaron cinco niveles de concentración (entre 0.1 y $25 \mu \mathrm{g} / \mathrm{L}$ ) de estándar de HAP (Supelco, Sigma). El coeficiente de determinación $\left(\mathrm{R}^{2}\right)$ para todos los compuestos fue superior a $0.9991(\mathrm{p}<0.005)$.

\section{Análisis estadístico}

Para el análisis de los cromatogramas se empleó el programa integrado Chromeleon 7.2.0.3765 (Dionex, Thermo Scientific). Los valores de HAP fueron referidos al volumen de aire filtrado (ng/L).

\section{Control de calidad}

A fin de determinar la eficiencia del método a través del rendimiento real de la extracción, se llevó a cabo un control de calidad empleando filtros con y sin partículas depositadas sobre su superficie. Para ello, los filtros fueron previamente lavados tres veces con DCM, secados en estufa a $60{ }^{\circ} \mathrm{C}$ y divididos en dos grupos: un conjunto fue reservado en la estufa (testigo) en tanto que el otro fue empleado para recolectar $\mathrm{MP}_{2.5}$ (con material). Posteriormente, ambos grupos fueron subdivididos en partes iguales, y se añadió, a un subgrupo, estándar para obtener una concentración de $25 \mu \mathrm{g} / \mathrm{L}$ en el volumen final de extracción de un $\mathrm{mL}$. Los grupos configurados resultantes fueron: 1) testigo, 2) testigo + estándar, 3) con material y 4) con material + estándar. Todos los filtros de todos los grupos fueron sometidos al proceso de extracción y medición detallados anteriormente. Se calcularon porcentajes de recuperación para cada caso y se consideró recuperación satisfactoria aquellos en los cuales dicho porcentaje fuera superior al $85 \%$.

\section{RESULTADOS}

Los valores de emisión y absorción óptimos teóricos para cada HAP, así como los eluyentes empleados se seleccionaron considerando reportes anteriores (Peltonen et al. 1995, Schauer et al. 2003, Dionex 2009, Szulejko et al. 2014). Luego estos valores se comprobaron experimentalmente empleando $25 \mu \mathrm{L}$ de estándar de HAP. Además, se determinaron experimentalmente el flujo óptimo de los eluyentes seleccionados (ACN grado HPLC y agua MilliQ) y el programa de la curva no isocrática. Con esta información se armó el programa final, que tiene una duración total de 25 minutos, temperatura de columna de $35^{\circ} \mathrm{C}$ y postcolumna de $30^{\circ} \mathrm{C}$, flujo de trabajo de $0.8 \mathrm{~mL} / \mathrm{min}$ y lectura UV constante a $254 \mathrm{~nm}$.

Cada muestra fue medida siete veces, de esta manera se pudo calcular un coeficiente de variación (CV) para cada compuesto.

Para determinar los tiempos de cambios de absorción y emisión de FL, se realizaron 45 corridas cromatográficas, primero, manteniendo constante el valor de absorción y variando el de emisión, y luego se realizó el proceso inverso. Los valores de absorción probados fueron: 230, 250, 270, $290 \mathrm{y}$ $310 \mathrm{~nm}$, en tanto que los de emisión: $320,350,380$, $395,410,425,440,470$ y $500 \mathrm{~nm}$. Con los datos de cuentas, se determinó la absorción/emisión máxima para cada compuesto, por ejemplo, para fenantreno (Fig. 2). Cada valor fue cotejado luego con los de

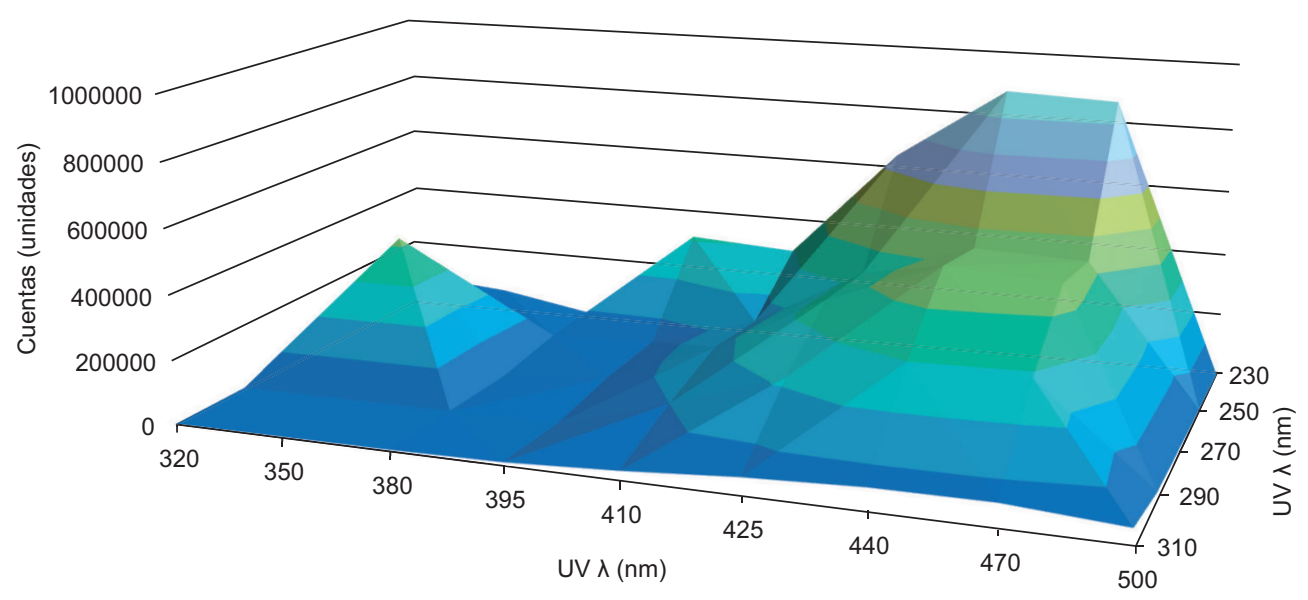

Fig. 2. Curva de emisión/absorción de fenantreno, utilizada para determinar los valores óptimos del compuesto para el armado del programa final 
CUADRO I. TIEMPOS DE RETENCIÓN DE LOS HIDROCARBUROS AROMÁTICOS POLICÍCLICOS MEDIDOS, CON SUS RESPECTIVAS LONGITUDES DE ABSORCIÓN Y EMISIÓN, LÍMITES DE DETECCIÓN (LOD) Y DE CUANTIFICACIÓN (LOQ), PORCENTAJE DE RECUPERACIÓN Y COEFICIENTE DE VARIABILIDAD $(\% \mathrm{CV})$

\begin{tabular}{lccccccc}
\hline HAP & $\begin{array}{c}\text { Tiempo de retención } \\
(\min \pm 0.150 \mathrm{~min})\end{array}$ & $\begin{array}{c}\text { Longitud de } \\
\text { absorción }(\lambda)\end{array}$ & $\begin{array}{c}\text { Longitud de } \\
\text { emisión }(\lambda)\end{array}$ & LOD & LOQ & $\begin{array}{c}\text { Recuperación } \\
(\%)\end{array}$ & $\begin{array}{c}\text { Variabilidad } \\
(\% \mathrm{CV})\end{array}$ \\
\hline Naf & 6.04 & 270 & 350 & 0.125 & 0.326 & 92.7 & 4.7 \\
Ace & 7.66 & 270 & 320 & 0.230 & 0.295 & 85.9 & 17.6 \\
Flu & 7.955 & 230 & 350 & 0.003 & 0.004 & 85.0 & 32.8 \\
Fen & 8.402 & 250 & 410 & 0.214 & 0.358 & 115.0 & 16.2 \\
Ant & 8.883 & 250 & 395 & 0.011 & 0.024 & 85.0 & 6.1 \\
Fla & 9.962 & 230 & 440 & 0.053 & 0.073 & 103.9 & 3.2 \\
Pir & 10.92 & 230 & 395 & 0.073 & 0.080 & 99.8 & 2.4 \\
B(a,h)a & 12.375 & 230 & 395 & 0.003 & 0.008 & 85.0 & 2.1 \\
Cri & 14.837 & 250 & 410 & 0.199 & 0.568 & 115.0 & 1.2 \\
B(b)f & 15.147 & 230 & 410 & 0.016 & 0.043 & 85.0 & 1.8 \\
B(k)f & 16.327 & 270 & 410 & 0.028 & 0.069 & 100.6 & 1.9 \\
B(a)pir & 16.863 & 230 & 410 & 0.053 & 0.138 & 85.0 & 3.4 \\
Dib(a,h)a & 18.947 & 250 & 470 & 0.040 & 0.067 & 86.3 & 2.3 \\
\hline
\end{tabular}

$\mathrm{HAP}=$ hidrocarburo aromático policíclico, $\mathrm{Naf}=$ naftaleno, Ace $=$ acenafteno, Flu $=$ fluoreno, Fen $=$ fenantreno, Ant $=$ antraceno, Fla = fluoranteno, $\mathrm{Pir}=$ pireno, $\mathrm{B}(\mathrm{a}, \mathrm{h}) \mathrm{a}=$ benzo(a,h)antraceno, Cri $=$ criseno, $\mathrm{B}(\mathrm{b}) \mathrm{f}=$ benzo(b)fluoranteno, $\mathrm{B}(\mathrm{k}) \mathrm{f}=$ benzo(k)fluoranteno, B(a)p = benzo(a)pireno, Dib(a,h)a = dibenzo(a,h)antraceno

los compuestos cuyas eluciones fueran próximas entre sí en el tiempo y se seleccionó una longitud de absorción y emisión óptimas para ese grupo de sustancias.

Los valores óptimos de emisión y absorción de cada compuesto, así como los límites de detección y cuantificación y los porcentajes de recuperación, pueden observarse en el cuadro I. Los cambios de eluyentes y tiempos de absorción/emisión finalmente empleados se presentan en la figura 3. Por otro lado, en el cuadro II se presentan resultados de algunas muestras medidas con el programa resultante. Es importante destacar que cuando las concentraciones de los compuestos medidos mostraban valores que se encontraban por sobre la curva de calibración, se realizaron diluciones seriadas 1:2 hasta obtener mediciones acordes a dicha curva.

En la figura 4 se observan dos corridas cromatográficas resultantes de la metodología propuesta.

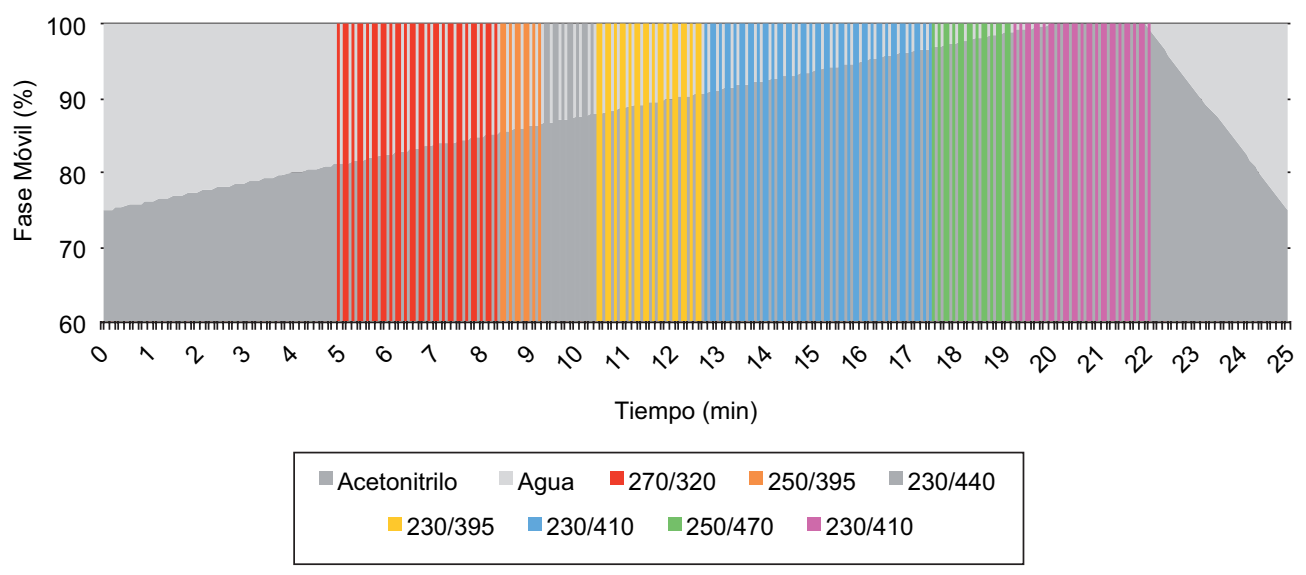

Fig. 3. Programa de corrida final utilizado para la detección y cuantificación de hidrocarburos aromáticos policíclicos mediante cromatografía líquida de alta eficiencia 
CUADRO II. CONCENTRACIÓN (ng/L) DE LOS HIDROCARBUROS AROMÁTICOS POLICÍCLICOS ANALIZADOS EN ALGUNAS DE LAS MUESTRAS EXAMINADAS

\begin{tabular}{|c|c|c|c|c|c|c|c|c|c|c|c|}
\hline \multirow{2}{*}{ HAP } & \multicolumn{11}{|c|}{ Muestras } \\
\hline & 1 & 2 & 3 & 4 & 5 & 6 & 7 & 8 & 9 & 10 & 11 \\
\hline $\mathrm{Naf}$ & 231.24 & 30.08 & 109.02 & 149.38 & 175.76 & 126.35 & 204.46 & 152.6 & 34.56 & 74.56 & 43.41 \\
\hline Ace & 40.31 & 6.51 & 14.97 & 22.17 & 9.65 & 18.96 & 42.94 & 42.01 & 9.66 & 16.41 & 13.78 \\
\hline Flu & 44.88 & 22.31 & 51.06 & 36.78 & 20.36 & 33.39 & 45.81 & 35.37 & 18.19 & 40.48 & 32.46 \\
\hline Fen & 155.36 & 136.34 & 279.58 & 186.41 & 313.51 & 158.47 & 155.11 & 101.89 & 97.84 & 155.84 & 142.53 \\
\hline Ant & 10.28 & 5.85 & 12.34 & 8.34 & 22.09 & 8.29 & 6.81 & 7.49 & 5.94 & 7.87 & 8.25 \\
\hline Fla & 80.74 & 32.84 & 88.33 & 54.8 & 92.39 & 49.57 & 44.61 & 38.83 & 38.6 & 47.31 & 41.24 \\
\hline Pir & 19.02 & 7.83 & 22.61 & 10.17 & 21.52 & 15.7 & 11.76 & 9.42 & 12.6 & 10.69 & 10.69 \\
\hline $\mathrm{B}(\mathrm{a}, \mathrm{h}) \mathrm{a}$ & 30.04 & 4.26 & 26.22 & 4.05 & 6.55 & 8.34 & 5.94 & 4.11 & 8.51 & 5.53 & 4.06 \\
\hline Cri & 11.48 & 0.55 & 6.01 & 0.36 & 34.43 & 16.27 & 0.14 & 0.25 & 30.6 & 0.45 & 3.47 \\
\hline$B(b) f$ & 3.13 & 0.45 & 1.45 & 0.34 & 13.38 & 9.38 & 0.2 & 0.27 & 17.57 & 0.42 & 2.11 \\
\hline $\mathrm{B}(\mathrm{k}) \mathrm{f}$ & 7.67 & 0.99 & 3.34 & 0.7 & 38.43 & 10.43 & 0.43 & 0.55 & 33.44 & 0.71 & 3.71 \\
\hline B(a)pir & 19.55 & 0.56 & 2.33 & 0.99 & 75.56 & 8.21 & 0.16 & 0.49 & 24.17 & 0.4 & 0.53 \\
\hline $\operatorname{Dib}(\mathrm{a}, \mathrm{h}) \mathrm{a}$ & 19.99 & 1.48 & 0.87 & 0.86 & 155.34 & 26.62 & 0.55 & 0.76 & 42.21 & 1.56 & 9.05 \\
\hline
\end{tabular}

$\mathrm{HAP}=$ hidrocarburo aromático policíclico, $\mathrm{Naf}=$ naftaleno, Ace $=$ acenafteno, Flu $=$ fluoreno, Fen $=$ fenantreno, Ant $=$ antraceno, $\mathrm{Fla}=$ fluoranteno, $\mathrm{Pir}=$ pireno, $\mathrm{B}(\mathrm{a}, \mathrm{h}) \mathrm{a}=$ benzo $(\mathrm{a}, \mathrm{h})$ antraceno, $\mathrm{Cri}=$ criseno, $\mathrm{B}(\mathrm{b}) \mathrm{f}=$ benzo(b)fluoranteno, $\mathrm{B}(\mathrm{k}) \mathrm{f}=$ benzo $(\mathrm{k})$ fluoranteno, $\mathrm{B}(\mathrm{a}) \mathrm{p}=$ benzo(a)pireno, Dib(a,h)a = dibenzo(a,h)antraceno
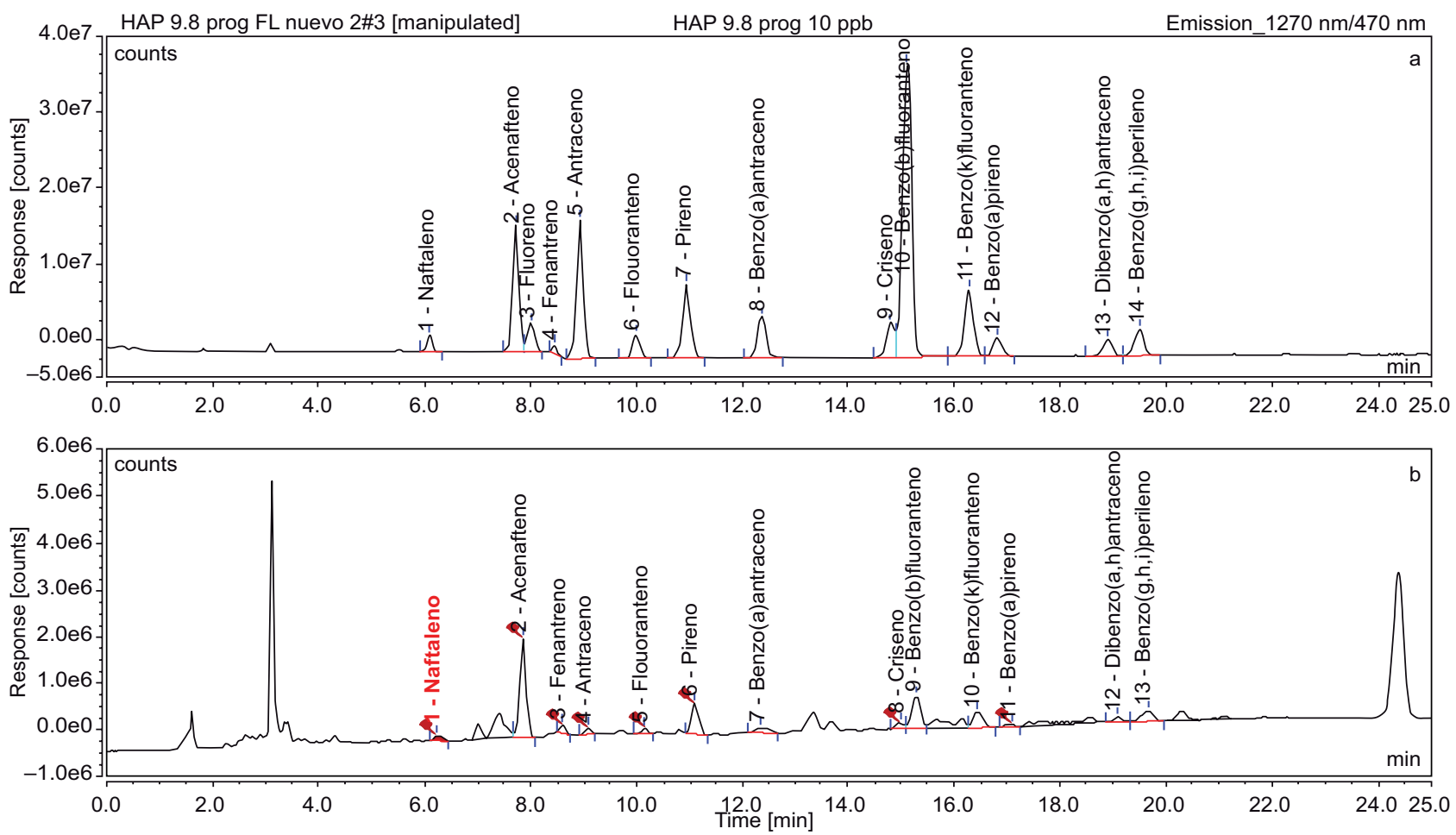

Fig. 4. Cromatogramas de un estándar de hidrocarburos aromáticos policíclicos (HAP), $10 \mathrm{ppb}$ (a) y de una muestra de partículas extraída con la metodología propuesta (b) 


\section{DISCUSIÓN}

En el cuadro III se comparan las características del método evaluado en este trabajo y el TO-13, que es el método de referencia recomendado por USEPA.

En el método desarrollado por USEPA, la extracción de los compuestos se realiza con Soxhlet, utilizando para ello $700 \mathrm{~mL}$ de disolvente (dietiléter:hexano 10:90 V/V) por cada muestra. Mientras que en la metodología propuesta sólo se utilizan $7 \pm 1 \mathrm{~mL}$ de DCM por muestra, con lo cual se reduce casi 100 veces la cantidad de disolvente utilizado para la extracción.

En cuanto a las características de los disolventes utilizados para la extracción, el método TO-13 propone extracciones con mezclas de dietiléter y hexano. El primero es un líquido inflamable y presenta un grave peligro de incendio. En exposiciones de corta duración irrita los ojos y el tracto respiratorio. La ingestión del líquido puede originar aspiración dentro de los pulmones con riesgo de neumonitis química. También puede causar efectos en el sistema nervioso central, dando lugar a narcosis (IPCS 2008). El hexano también es un disolvente altamente inflamable que irrita la piel en exposiciones de corta duración. Además, la ingestión del líquido puede originar aspiración dentro de los pulmones con riesgo de neumonitis química. La exposición a altas concentraciones podría causar disminución del estado de alerta (IPCS 2006). En este caso, al emplear dos sustancias altamente inflamables, hay que tener especial cuidado con la temperatura de trabajo por el riesgo de incendio. Esto es particularmente importante, considerando las altas cantidades de disolvente utilizado con este método, por lo que las probabilidades de accidentes aumentan proporcionalmente. Con la nueva metodología propuesta, se emplea DCM que es un depresor del sistema nervioso central y un posible carcinógeno para el humano según el Centro Internacional de Investigaciones sobre el Cáncer (IARC 1999). En exposiciones de corta duración, la sustancia irrita los ojos, la piel y el tracto respiratorio, y también podría causar disminución del estado de alerta (IPCS 2000). Si bien este disolvente también presenta efectos adversos, las bajas cantidades que se emplean minimizan los riesgos de exposición, más aún si se trabaja con un recuperador de disolvente bajo campana.

En el método planteado por USEPA, el tiempo de extracción Soxhlet es de $18 \mathrm{~h}$ por extracción. En cambio, el método planteado requiere de 6 a $8 \mathrm{~h}$ totales por cada 10 muestras. Si bien el trabajo es más manual que en el método TO-13, los tiempos requeridos para extraer los compuestos son visiblemente menores. A esto se suma que el tiempo de corrida de cada muestra es la mitad que aquel detallado en el método de USEPA. Como resultado se reducen también los costos de mano de obra requerida.

Otra diferencia importante entre los métodos mencionados está relacionada con el procedimiento de cuantificación de los HAP. Se utiliza cromatografía líquida y no gaseosa. Hace un par de décadas, se

CUADRO III. COMPARACIÓN DE LA METODOLOGÍA PROPUESTA PARA EXTRACCIÓN DE HIDROCARBUROS AROMÁTICOS POLICÍCLICOS CON LA TO-13 DE LA AGENCIA DE PROTECCIÓN AMBIENTAL DE ESTADOS UNIDOS

\begin{tabular}{lll}
\hline Características & Este estudio & TO-13 \\
\hline Volumen de aire muestreado $(24 \mathrm{~h})$ & $18 \pm 0.71 \mathrm{~m}^{3}$ & $300 \mathrm{~m}^{3}$ \\
Sistema de extracción de HAP & Tubos de reflujo cerrado & Soxhlet \\
Solvente de extracción & Diclorometano grado HPLC & Dietiléter/Hexano \\
Enrase final de muestra & $1 \mathrm{~mL}$ & $1 \mathrm{~mL}$ \\
Equipo de cromatografía & $\mathrm{HPLC}$ & $\mathrm{GC}$ \\
Sistema de detección & $\mathrm{FL}$ & $\mathrm{MS}$ \\
Calibración con estándar & 5 puntos & $5 \mathrm{puntos}$ \\
& $(0.0001,0.001,0.005,0.010$ y $0.025 \mathrm{ppm})$ & $(0.10,0.25,0.50,1.25$ y $2.50 \mathrm{ppm})$ \\
Temperatura de trabajo & $35^{\circ} \mathrm{C}$ & $250-300{ }^{\circ} \mathrm{C}$ \\
Volumen de inyección de muestra & $20 \mu \mathrm{L}$ & $2 \mu \mathrm{L}$ \\
Columna & $\mathrm{C}-18$ de silica, fase reversa, $250 \mathrm{~mm}$ x 2.1 I.D., & DB-5 de silica, $30 \mathrm{~m} \mathrm{x} 0.32 \mathrm{~mm}$ I.D. \\
& tamaño de empaque $5 \mu \mathrm{m}$ & \\
Acarreador & Acetonitrilo grado HPLC + MilliQ & Helio ultra alta pureza \\
Tiempo total de corrida & 25 min & Aproximadamente 50 min \\
Desvío de tiempo en detección de picos & 0.15 min & 0.10 min \\
Sensibilidad de detección & ng & $\mu \mathrm{g}$ \\
\hline
\end{tabular}


discutía la utilización de HPLC como técnica para la detección de HAP debido a la baja resolución que poseían los equipos. Sin embargo, con los aparatos modernos se ha mejorado visiblemente su resolución, empleando detectores de FL, que son más sensibles que los que se empleaban anteriormente en los equipos de HPLC (luz UV) (por ejemplo: Dong et al. 1976, Krstulovic et al. 1976). De hecho, los nuevos equipos de cromatografía líquida superan en sensibilidad a los aparatos GC, aunque esto depende fuertemente de las características del equipo empleado y de la columna utilizada (Gratz et al. 2000, Liaud et al. 2015). Otras ventajas adicionales del uso de HPLC en vez de GC es que se pueden emplear acarreadores más económicos y de fácil manipulación, temperaturas de trabajo más bajas y columnas de separación más versátiles.

Estudios previos de intercalibración entre GC y HPLC, encontraron mayores tiempos de análisis, menor resolución, menor límite de detección (LOD) e incluso algunos picos de compuestos solapados en las muestras corridas en cromatografía de gases (Chiu et al. 1997).

Los métodos GC/MS capilares se limitan al análisis de aquellos HAP que son volátiles y térmicamente estables, mientras que un sistema de detección de FL supera el problema de la resolución en HPLC por el hecho de que dos longitudes de onda, excitación y emisión, están disponibles para la medición. Por lo tanto, aunque dos o más compuestos en una mezcla de HAP multicomponente pueden tener la misma longitud de onda de excitación para emitir fluorescencia, es poco probable que también emitan a la misma longitud de onda. Además, un HAP individual puede tener más de una longitud de onda fluorescente en la que puede excitarse. Por lo tanto, mediante la selección cuidadosa de las longitudes de onda de excitación y emisión, se puede obtener un alto grado de especificidad y selectividad en la detección de fluorescencia (Das y Thomas 1978).

\section{CONCLUSIONES}

Las modificaciones introducidas en la metodología generaron reducciones tanto en los tiempos, como en los costos de procesamiento, detección y cuantificación de HAP adsorbidos a partículas en suspensión.

Esto implica una mejora sustancial en el tiempo de procesamiento de cada muestra, sin reducir la sensibilidad para la detección de compuestos. Por otro lado, hay una disminución considerable en la cantidad de disolvente utilizado, con lo cual resulta en una metodología menos costosa y más amigable con el ambiente.

\section{REFERENCIAS}

Carreras H.A., Calderón-Segura M.E., Gómez-Arroyo S., Murillo-Tovar M.A. y Amador-Muñoz O. (2013). Composition and mutagenicity of PAHs associated with urban airborne particles in Córdoba, Argentina. Environ. Pollut. 178, 403-410.

DOI: 10.1016/j.envpol.2013.03.016

Chiu C.P., Lin Y.S. y Chen B.H. (1997). Comparison of GC-MS and HPLC for overcoming matrix interferences in the analysis of PAHs in smoked food. Chromatographia 44 (9-10), 497-504.

DOI: $10.1007 / \mathrm{BF} 02466743$

Dionex (2009). Determination of polycyclic aromatic hydrocarbons (PAHs) in tap water using on-line solid-phase extraction followed by HPLC with UV and fluorescence detections. Corporación Dionex. Nota de aplicación 213, LPN 2128. Sunnyvale, California, EUA, 8 pp.

Dong M., Locke D.C. y Ferrand E. (1976). High pressure liquid chromatographic method for routine analysis of major parent polycyclic aromatic hydrocarbons in suspended particulate matter. Anal. Chem. 48 (2), 368-372. DOI: 10.1021/ac60366a049

Gratz L.D., Bagley S.T., Leddy D.G., Johnson J.H., Chiu C. y Stommel P. (2000). Interlaboratory comparison of HPLC-fluorescence detection and GC/MS: analysis of PAH compounds present in diesel exhaust. J. Hazard Mater. 74 (1-2), 37-46.

DOI: 10.1016/S0304-3894(99)00197-1

IARC (1999). Dichloromethane. IARC Monographs on the Evaluation of Carcinogenic Risks to Humans. International Agency for Research on Cancer. Monografía. Lyon, Francia, 66 pp.

IPCS (2000). Ficha Internacional de Seguridad Química 0058, Diclorometano. International Programme on Chemical Safety. Organización Mundial de la Salud, abril de 2017.

IPCS (2008). Ficha Internacional de Seguridad Química 0355. Dietil éter. International Programme on Chemical Safety. Organización Mundial de la Salud, mayo de 2002.

IPCS (2006). Ficha Internacional de Seguridad Química 0279. Hexano. International Programme on Chemical Safety. Organización Mundial de la Salud, abril de 2000.

Kim K.H., Jahan S.A., Kabir E. y Brown R.J. (2013). A review of airborne polycyclic aromatic hydrocarbons (PAHs) and their human health effects. Environ Int. 60, 71-80. DOI: 10.1016/j.envint.2013.07.019 
Krstulovic A.M., Rosie D.M. y Brown P.R. (1976). Selective monitoring of polynuclear aromatic hydrocarbons by high pressure liquid chromatography with a variable wavelength detector. Anal. Chem. 48 (9), 1383-1386. DOI: $10.1021 / \mathrm{ac50003a029}$

Liaud C., Millet M. y Le Calvé S. (2015). An analytical method coupling accelerated solvent extraction and HPLC-fluorescence for the quantification of particlebound PAHs in indoor air sampled with a 3-stages cascade impactor. Talanta 131, 386-394.

DOI: 10.1016/j.talanta.2014.05.027

López M.L., Ceppi S., Palancar G.G., Olcese L.E., Tirao G. y Toselli B.M. (2011). Elemental concentration and source identification of PM10 and PM2.5 by SR-XRF in Córdoba city, Argentina. Atmos. Environ. 45 (31), 5450-5457. DOI: 10.1016/j.atmosenv.2011.07.003

Masih J., Singhvi R., Kumar K., Jain V.K. y Taneja A. (2012). Seasonal variation and sources of polycyclic aromatic hydrocarbons (PAHs) in indoor and outdoor air in a semi-arid tract of northern India. Aerosol Air Qual. Res. 12, 515-525.

DOI: 10.4209/aaqr.2011.11.0192

Mateos A.C., Amarillo A.C., Carreras H.A., y González C.M. (2018). Land use and air quality in urban environments: human health risk assessment due to inhalation of airborne particles. Environ. Res. 161, 370-380.

DOI: 10.1016/j.envres.2017.11.035

Peltonen K. y Kuljukka T. (1995). Air sampling and analysis of polycyclic aromatic hydrocarbons. J. Chromatogr. A. 710 (1), 93-108.

DOI: $10.1016 / 0021-9673(95) 00207-4$
Schauer C., Niessner R. y Pöschl U. (2003). Polycyclic aromatic hydrocarbons in urban air particulate matter: decadal and seasonal trends, chemical degradation, and sampling artifacts. Environ. Sci. Technol. 37 (13), 2861-2868. DOI: $10.1021 / \mathrm{es} 034059 \mathrm{~s}$

Stein A.F. y Toselli B.M. (1996). Street level air pollution in Córdoba city, Argentina. Atmos. Environ. 30 (20), 3491-3495. DOI: 10.1016/1352-2310(96)00097-0

Szulejko J.E., Kim K.H., Brown R.J. y Bae M.S. (2014). Review of progress in solvent-extraction techniques for the determination of polyaromatic hydrocarbons as airborne pollutants. Trac-Trend Anal. Chem. 61, 40-48. DOI: 10.1016/j.trac.2014.07.001

Tsai J.H., Being-Hwa P., Ding-Zang L. y Lee C.C. (1995). PAH characteristics and genotoxicity in the ambient air of a petrochemical industry complex. Environ. Int. 21 (1), 47-56. DOI: 10.1016/0160-4120(94)00037-8

USEPA (1996). Compendium of methods for the determination of toxic organic chemicals in ambient air. Reporte No. EPA/600/R-96/010b. Agencia de Protección Ambiental de Estados Unidos. Reporte. Cincinnati, Ohio, EUA, 84 pp.

USEPA (2003). Baseline human health risk assessment for the Ogden Railyard site Ogden, Utah. Agencia de Protección Ambiental de Estados Unidos. Investigación. Denver, Colorado, EUA, 383 pp.

WHO (2003). Polynuclear aromatic hydrocarbons in drinking-water. Background document for development of WHO guidelines for drinking-water quality. Organización Mundial de la Salud. Documento de antecedentes. Geneva, Suiza, 32 pp. 